\title{
Motion Planning for Mobile Manipulators along Given End-effector Paths
}

\author{
Giuseppe Oriolo Christian Mongillo \\ Dipartimento di Informatica e Sistemistica \\ Università di Roma "La Sapienza" \\ Via Eudossiana 18, 00184 Roma, Italy \\ oriolo@dis.uniroma1.it,chrismongillo@infinito.it
}

\begin{abstract}
We consider the problem of planning collisionfree motions for a mobile manipulator whose end-effector must travel along a given path. Algorithmic solutions are devised by adapting a technique developed for fixed-base redundant robots. In particular, we exploit the natural partition of generalized coordinates between the manipulator and the mobile base, whose nonholonomy is accounted for at the planning stage. The approach is based on the randomized generation of configurations that are compatible with the endeffector path constraint. The performance of the proposed algorithms is illustrated by several planning experiments.

Index Terms-Mobile manipulators, probabilistic motion planning.
\end{abstract}

\section{INTRODUCTION}

Mobile manipulators combine the two archetypes of robotic systems, i.e., articulated arms and mobile platforms: hence, they exhibit the dexterity and grasping capability of the former and the sensor-based mobility of the latter. Several prototypes of mobile manipulators already exist; see [1-2] for some examples. However, many research aspects still need to be addressed in order to fully exploit the potential of these mechanisms. In fact, the arm and the mobile platform are often treated as distinct entities, neglecting their kinematic and dynamic interaction, whereas their most effective use is expected to rely on the coordinated use of locomotion and manipulation functions [3].

Planning collision-free motions under task constraints is a typical problem where whole-system coordination is crucial. In many applications, the mobile manipulator is required to move the end-effector along a given path in order to realize the task specified by a higher-level module (e.g., for inspection missions with an in-hand camera, or in pick and place operations). A lower-level planner is then in charge of generating joint paths that realize the desired endeffector motion while guaranteeing that the robot avoids collisions with obstacles or with itself. We call this problem Motion Planning along End-effector Paths (MPEP).

Since mobile manipulators are kinematically redundant with respect to end-effector tasks, the MPEP problem can be attacked as an optimal redundancy resolution problem, with the additional difficulty that the mobile platform is often subject to nonholonomic constraints; one possibility is therefore to adapt kinematic [4] or optimal [5] control schemes. However, none of the above solutions is satisfactory when the objective is obstacle avoidance. For example, the optimal control formulation of the MPEP problem for mobile manipulators proposed in [6] leads to a nonlinear TPBVP whose solution can only be sought numerically, without any guarantee of success.

The objective of this paper is to present a family of probabilistic planners for solving the MPEP problem in mobile manipulators by extending our previous work dealing with the same problem in fixed-base redundant manipulators [7]. We exploit the natural partition of generalized coordinates between the manipulator and the mobile platform, and take into account the presence of nonholonomic constraints at the planning stage. All the planners rely on the same mechanism for generating random configurations that are compatible with the end-effector constraint.

The paper is organized as follows. In the next section, we give a precise formulation of the MPEP problem for mobile manipulators and clarify what we consider to be a solution. The procedure for generating random configurations is then presented, and the various proposed planners are described. Results for problems of increasing complexity are finally presented to illustrate the performance of the algorithms.

\section{MPEP PROBLEM FOR MOBILE MANIPULATORS}

In this section, we generalize our formulation of the MPEP problem [7] so as to apply to the mobile manipulator case. With respect to fixed-base manipulators, the essential features of mobile manipulators are the natural partition of generalized coordinates (mobile platform/manipulator) and the nonholonomy due to the rolling wheels.

Consider a mobile manipulator whose task is to move the end-effector along a given path in a workspace populated by obstacles. The direct kinematics is expressed as

$$
p=f(q)=f\left(\begin{array}{c}
q^{p} \\
q^{m}
\end{array}\right),
$$

where $p \in \mathbb{R}^{M}$ is the end-effector pose (position and/or orientation) and $q \in \mathbb{R}^{N}$ is the system configuration ${ }^{1}$, consisting of the platform configuration $q^{p} \in \mathbb{R}^{N_{p}}$ and the manipulator configuration $q^{m} \in \mathbb{R}^{N_{m}}$, with $N_{p}+N_{m}=N$. While the manipulator subsystem is holonomic (i.e., arbitrary motions are possible for the manipulator configuration $\left.q^{m}\right)$, the motion of the platform is generated as

$$
\dot{q}^{p}=G\left(q^{p}\right) u,
$$

${ }^{1}$ We consider euclidean spaces for simplicity, but our developments apply to the case in which $p, q^{p}$ and $q^{m}$ are defined over manifolds. 
where $u \in \mathbb{R}^{P}$ are pseudovelocities (typically, linear and angular platform velocities), while the columns of $G\left(q^{p}\right)$ span the null space of the nonholonomic constraint matrix.

An end-effector path $p(\sigma)$ is assigned, with $\sigma \in[0,1]$ the path parameter. For the problem to be well-posed, we assume that $\forall \sigma \in[0,1], p(\sigma) \in \mathcal{T}$, where $\mathcal{T} \subset \mathbb{R}^{M}$ is the dextrous task space, defined as the set of end-effector poses that can be realized by $\infty^{N-M}$ configurations ${ }^{2}$.

Assume that the mobile manipulator is kinematically redundant with respect to the given task, i.e., $N>M$. Then, the MPEP problem is to find a configuration path $q(\sigma)=\left(q^{p}(\sigma), q^{m}(\sigma)\right)$ such that:

1) $p(\sigma)=f(q(\sigma)), \forall \sigma \in[0,1]$;

2) the robot does not collide with obstacles or itself;

3) the path is feasible w.r.t. the kinematic constraints that may exist (e.g., manipulator joint limits);

4) the path is feasible w.r.t. the nonholonomic constraints, i.e., $q^{p}(\sigma)$ is a solution of eq. (2).

Depending on the application, an initial joint configuration $q(0)$ such that $p(0)=f(q(0))$ may or not be assigned. The first version of the problem is more constrained (and thus possibly easier to solve) than the second.

We seek a solution to the MPEP problem in the form of a sequence of configurations:

$$
\left\{q\left(\sigma_{0}\right), q\left(\sigma_{1}\right), \ldots, q\left(\sigma_{s-1}\right), q\left(\sigma_{s}\right)\right\}, \quad \sigma_{0}=0, \sigma_{s}=1,
$$

with the $\sigma_{i}$ 's equispaced and $p\left(\sigma_{i}\right)=f\left(q\left(\sigma_{i}\right)\right)$. The integer $s$ is called path sampling. A continuous path will be derived from this sequence by joining successive configurations by a local planner; this may use simple linear interpolation ${ }^{3}$ for $q^{m}$, while the mobile platform nonholonomy must be taken into account when joining successive values of $q^{p}$.

\section{Generation OF RANDOM CONFigurations}

The algorithms we have developed for solving the MPEP problem share the same basic tool, i.e., a procedure which performs random sampling of self-motion manifolds. The mechanism proposed in [7] for generating $N$-dimensional random configurations compatible with the $M$-dimensional task constraint is based on a partition of $q$ into $M$ base and $N-M$ redundant variables; the value of the latter is first randomly generated, and the value of base variables is then computed by inverse kinematics in such a way that the resulting configuration places the end-effector at a certain point of its assigned path ${ }^{4}$. In this section, we show how this basic strategy can be adapted to mobile manipulators.

Assume for illustration that the platform is a unicycle, described by coordinates $x, y, \theta$ (position and orientation) and controlled by pseudovelocities $v, \omega$ (linear and angular velocity), while the manipulator is a spatial three-dof arm with rotational joints (Fig. 1). Hence, we have $q^{p} \in \mathbb{R}^{3}$, $q^{m} \in \mathbb{R}^{3}$, and $q \in \mathbb{R}^{6}$. The end-effector task is specified

${ }^{2} \mathcal{T}$ does not contain its boundary, which includes the unavoidable singularities realized by a single configuration.

${ }^{3}$ This will lead to an path error between successive poses, whose entity can however be reduced at will by increasing the sampling $s$.

${ }^{4}$ This sampling mechanism is similar to the one used in [8] for guaranteeing the closure constraint.

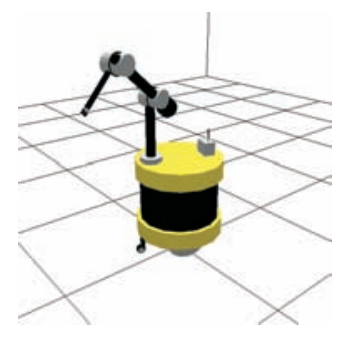

Fig. 1. The mobile manipulator considered in this paper

at the position level, i.e., $p \in \mathbb{R}^{3}$. The extension to the case where orientation is also specified is straightforward (provided that the manipulator has sufficient dof's).

As the configuration $q$ is naturally split between platform variables $q^{p}$ and manipulator variables $q^{m}$, it is reasonable to choose the base/redundant partition accordingly. Since in our case $M=3$ and $N=6$, we must select three variables as redundant, and generate their values randomly. Throughout the paper, we use the platform variables for this purpose. Other possibilities (e.g., using the manipulator variables or a mixed set) are not discussed here.

With the assumptions of Sect. II, each pose $p\left(\sigma_{i}\right) \in \mathcal{T}$ along the given end-effector path can be realized by $\infty^{N-M}=\infty^{3}$ configurations of the mobile manipulator, which represent the so-called self-motion manifold ${ }^{5}$. Assume that the configuration $q_{i}^{p}$ of the platform is randomly chosen. For each value of $p_{i}=p\left(\sigma_{i}\right), i=0, \ldots, s$, there exist a finite number (up to 4 , in our case) of manipulator configurations $q_{i}^{m}=q^{m}\left(p_{i}, q_{i}^{p}\right)$ such that $p_{i}=f\left(q_{i}^{p}, q_{i}^{m}\right)$, computed by inverting the kinematic map (1) with $q^{p}=q_{i}^{p}$. Depending on the value of $q_{i}^{p}$, it may happen that no value of $q_{i}^{m}$ is compatible with the end-effector pose $p_{i}$.

According to the above strategy, the procedure generating a random sample of the self-motion manifold corresponding to $p_{i}$ is described in pseudocode as follows:

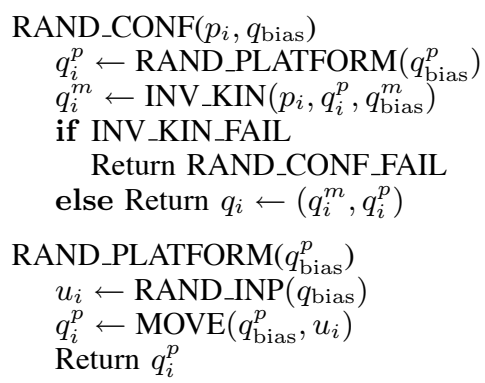

The effect of the optional argument $q_{\text {bias }}$, which appears in both procedures, is to bias the distribution characterizing the randomly generated samples. When $q_{\text {bias }}$ is present, RAND_CONF returns (if successful) a configuration $q_{i}$ such that $(i)$ there is a simple feasible path connecting $q_{\mathrm{bias}}^{p}$ to $q_{i}^{p}$, (ii) $p_{i}=f\left(q_{i}\right)$, and (iii) $\left\|q_{i}^{m}-q_{\text {bias }}^{m}\right\|_{\infty}<d$, where $d$ is a maximum allowed joint displacement. The first of these properties is guaranteed by RAND_PLATFORM, which generates a random pseudovelocity vector $u_{i}$ and then computes (by forward integration of eq. (2)) the platform

\footnotetext{
${ }^{5}$ To be precise, the inverse image of any point $p \in \mathcal{T}$ is in general a finite number of disjoint manifolds.
} 
configuration $q_{i}^{p}$ reached from $q_{\text {bias }}^{p}$ by applying $u_{i}$ over a sampling interval. The last two properties are enforced by INV_KIN, which takes as inputs $p_{i}$ and $q_{i}^{p}$ and seeks an inverse solution $q_{i}^{m}$ for the manipulator joints which also satisfies the displacement constraint. If no such solution exists (either because no inverse solution exists for the chosen $q_{i}^{p}$ or because all solutions violate the displacement constraint) the boolean INV_KIN_FAIL becomes true.

If $q_{\text {bias }}$ is absent, RAND_PLATFORM simply generates a random configuration $q_{i}^{p}$; hence, the self-motion manifold sample computed by RAND_CONF is not biased by any configuration. Finally, when RAND_CONF is invoked with no argument, a completely random configuration (not belonging to any self-motion manifold) is produced.

The reason for biasing the random generation of $q_{i}$ with $q_{\text {bias }}$ is that all the algorithms to be presented work in an incremental fashion, trying to build a connectivity roadmap from the initial end-effector pose. When a sample $q_{i}$ has been randomly generated on the self-motion manifold corresponding to $p_{i}$, it is used as $q_{\text {bias }}$ for the next selfmotion manifold in order to guarantee that $(i) q_{i}^{p}$ and $q_{i+1}^{p}$ are connected by a feasible path, and (ii) $q_{i+1}^{m}$ will be sufficiently close to $q_{i}^{m}$. The latter fact will ensure that the end-effector constraint violation between $q_{i}$ and $q_{i+1}$ due to the use of linear interpolation for $q^{m}$ is reduced.

We now give more details about two important issues.

\section{A. The Compatible Platform Region}

Given an end-effector pose $p_{i}$, it would be inefficient to generate the random sample $q_{i}^{p}$ (i.e., the mobile platform configuration) in the whole configuration space. The condition for the existence of an inverse kinematic solution $q_{i}^{m}$ for the manipulator is that $q_{i}^{p}$ 'places' the manipulator workspace so as to contain $p_{i}$. We could also say that $q_{i}^{p}$ must 'belong' to the self-motion manifold of $p_{i}$.

For simplicity, we shall use a coarse estimation of this manifold, based on the geometric argument in Fig. 2. With the manipulator in full extension (elbow singularity), a circle is drawn with the center at $p_{i}$ and passing through the platform center. Such circle (shown in gray) identifies the platform positions that we consider to be compatible with the end-effector constraint. Therefore, randomly generated $q_{i}^{p}$, s placing the platform outside this region are discarded.

Note that, even if $q_{i}^{p}$ is such that the platform position lies in the circle, there is no guarantee that an inverse kinematic solution $q_{i}^{m}$ exists. This will depend on the orientation component of $q_{i}^{p}$ as well as on the vertical component of $p_{i}$. However, the number of failures of INV_KIN using the above approximation of the self-motion manifold was found to be very low and therefore acceptable ${ }^{6}$.

If RAND_PLATFORM is called without a $q_{\text {bias }}$, the position part of $q_{i}^{p}$ is directly generated inside the compatible region. Finally, if RAND_PLATFORM is called without any argument (i.e., without a particular end-effector pose $p_{i}$ ), the compatible region is assumed to be the union of all the circles centered on the given end-effector path.

${ }^{6} \mathrm{~A}$ more accurate computation is possible following the ideas in [9].

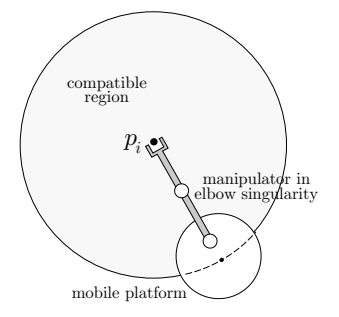

Fig. 2. Construction of the compatible platform region

\section{B. Generation of Random Pseudovelocities}

In practice, the pseudovelocities of the platform will be bounded. Let $\mathcal{U}=\mathcal{V} \times \Omega=\left[v_{\min }, v_{\max }\right] \times\left[\omega_{\min }, \omega_{\max }\right]$ be the set of admissible pseudovelocities.

The procedure RAND_INP for generating random pseudovelocity inputs is crucial, as it is responsible for the platform motion and, ultimately, for the effectiveness of the solution space exploration. In the remainder of this section, we discuss the different strategies developed to such end.

1) Completely random pseudovelocities: $v, \omega$ are generated according to a uniform probability distribution in $\mathcal{U}$.

2) Constant-energy pseudovelocities: $v$ is generated according to a uniform probability distribution in $\mathcal{V}$, while $\omega$ is computed from the following equation:

$$
v^{2}+c \omega^{2}=\gamma^{2}
$$

where $c>0$ is a weighting factor and $\gamma^{2}$ is the desired input energy level.

3) Optimal pseudovelocities: $v$ and $\omega$ are chosen among a set $\mathcal{C}$ of candidate pseudovelocities so as to optimize a certain criterion. In particular, $\mathcal{C}$ consists of four pseudovelocity vectors, randomly chosen within the subregions

$$
\begin{array}{ll}
\mathcal{U}_{1}=\left[0, v_{\max }\right] \times\left[0, \omega_{\max }\right] & \text { (a forward-right motion) } \\
\mathcal{U}_{2}=\left[0, v_{\max }\right] \times\left[\omega_{\min }, 0\right] & \text { (a forward-left motion) } \\
\mathcal{U}_{3}=\left[v_{\min }, 0\right] \times\left[\omega_{\min }, 0\right] & \text { (a backward-right motion) } \\
\mathcal{U}_{4}=\left[v_{\min }, 0\right] \times\left[0, \omega_{\max }\right] & \text { (a backward-left motion) }
\end{array}
$$

These four vectors may be generated as either completely random or constant-energy within their domain. We propose two different choices for the optimality criterion: the first (to be minimized) is $I_{\text {dist }}=\left\|q_{\text {des }}-q_{\text {new }}\right\|$, i.e., the (weighted) euclidean distance between the new configuration $q_{\text {new }}$ (obtained by moving the platform from $q_{\text {bias }}^{p}$ with the candidate pseudovelocity and then computing the manipulator posture through inverse kinematics) and a desired configuration $q_{\text {des }}$ (whose role will be clarified later, see Sect. IV-B). The second criterion $I_{\text {comp }}$ (to be maximized) is the task compatibility [10] of $q_{\text {new }}$, which quantifies the motion capability of the mobile manipulator along the end-effector path starting from the posture $q_{\text {new }}$.

The different effects of the above strategies will be illustrated in the planning experiments of Sect. V.

\section{Planning Algorithms}

Having discussed the procedure for generating random samples of a desired self-motion manifold, we now present the algorithms developed for the solution of the MPEP 
problem for mobile manipulators. All of them make use of the collision checking procedure NO_COLL. When invoked with a single argument $q_{i}$, it performs a collision check (including self-collisions) and returns true if $q_{i}$ is safe. When invoked with two arguments $\left(q_{i}, q_{j}\right)$, it performs a collision check on both configurations as well as on the path joining them (by sampling it at a sufficiently high rate). In particular, a feasible path (produced under the action of the constant pseudovelocity inputs selected by RAND_INP) is used for for the mobile platform, while the manipulator path is obtained by linear interpolation.

\section{A. Greedy Planner}

The core of the first algorithm is the STEP function which, given two generic poses $p_{i}, p_{k}(0 \leq i<k \leq s)$ belonging to the end-effector sequence and a configuration $q_{i}$ on the self-motion manifold of $p_{i}$, builds a (sub)sequence of configurations $\left\{q_{i}, \ldots, q_{k}\right\}$ connecting $p_{i}$ to $p_{k}$ and such that collisions are avoided along the path. If successful, STEP returns the sequence in the variable PATH.

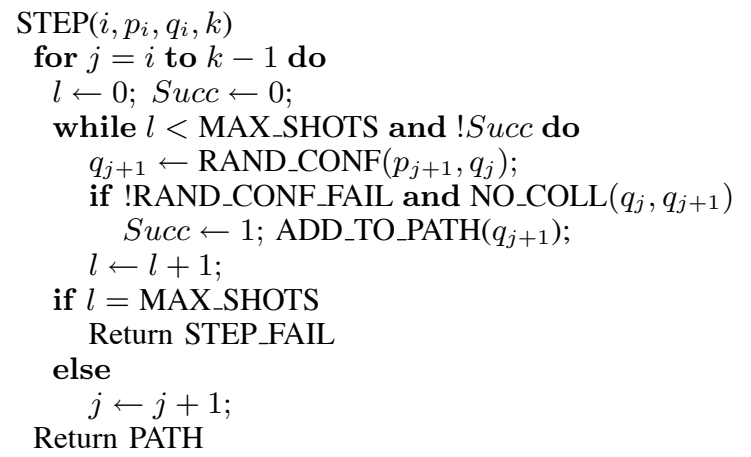

The parameter MAX_SHOTS represents the upper bound to the number of calls to RAND_CONF $\left(p_{j+1}, q_{j}\right)$ for each end-effector pose $p_{j}$. If RAND_CONF succeeds in finding a configuration $q_{j+1}$ realizing $p_{j+1}$, sufficiently close to the bias configuration $q_{j}$, and such that the path between $q_{j}^{p}$ and $q_{j+1}^{p}$ is feasible, the whole path between $q_{j}$ and $q_{j+1}$ is verified to be collision-free; in this case, $q_{j+1}$ is added to the current sequence through the ADD_TO_PATH function. If the maximum number of trials of RAND_CONF is exceeded, the procedure returns STEP_FAIL.

A direct approach to the solution is to devise a greedy algorithm based on iterated calls to the STEP function with $p_{0}, p_{s}$ as subsequence extrema and random $q_{0}$.

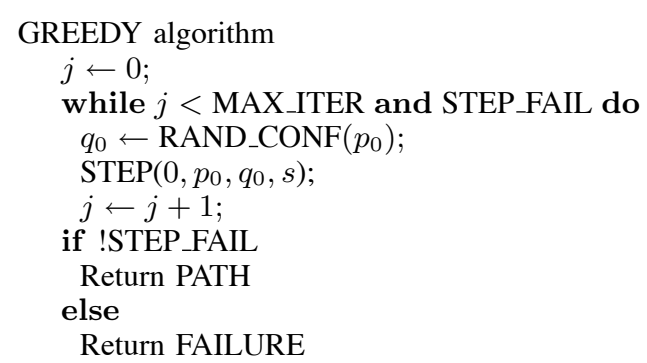

Given the initial pose $p_{0}$, RAND_CONF $\left(p_{0}\right)$ generates an initial configuration $q_{0}$ as described in the previous section. STEP is then invoked to search for a sequence of configurations guaranteeing feasible collision-free motion while the end-effector moves from $p_{0}$ to $p_{s}$. In case of success, the path found by STEP is returned. If STEP fails and MAX_ITER has not been exceeded, a new $q_{0}$ is generated and STEP starts a new search from $q_{0}$.

GREEDY implements a depth-first search, as for any initial configuration $q_{0}$ a sequence of random configurations (one for each self-motion manifold, and each biased by the previous one) is generated, and discarded if STEP does not reach the last self-motion manifold. Experiments have shown that this planner is effective in easy problems (see Sect. V), essentially due to the end-effector path constraint, which greatly reduces the admissible internal motions of the robot once a $q_{0}$ has been chosen. Still, the only possible way to backtrack for this planner is to generate a new $q_{0}$, and this may prove inefficient in complex problems.

\section{B. RRT-Like Planner}

To overcome the limitations of the depth-first algorithm GREEDY, one may try to generate multiple random samples for each self-motion manifold and to connect configurations on successive manifolds by local paths. As in [7], this exploratory behavior is achieved by the RRT_LIKE algorithm, which adapts the notion of RRT (Rapidlyexploring Random Tree, [11]) to mobile manipulators.

Our algorithm tries to expand a tree $\tau$ rooted at $q_{0}$, a random sample of the $p_{0}$ self-motion manifold, until the self-motion manifold of $p_{s}$ is reached. If the expansion fails a certain number of times, a different $q_{0}$ is generated and another tree is built, until the maximum number of iterations is exceeded. If a tree connecting $p_{0}$ to $p_{s}$ is found, a path is extracted by graph search techniques.

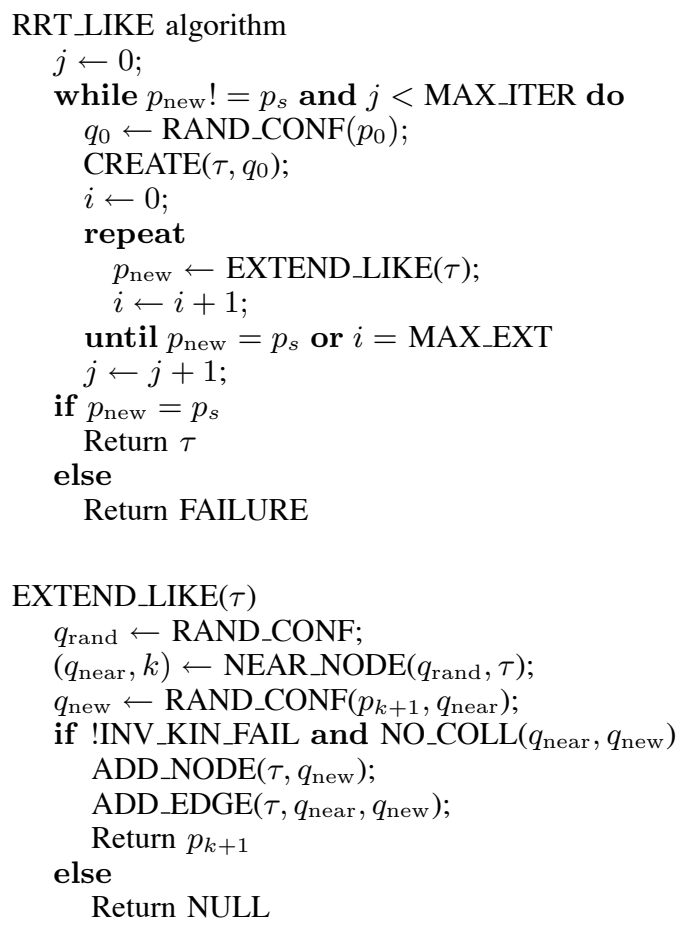

First, RAND_CONF is called with no arguments to find a random $q_{\text {rand }}=\left(q_{\text {rand }}^{p}, q_{\text {rand }}^{m}\right)$, and NEAR_NODE 


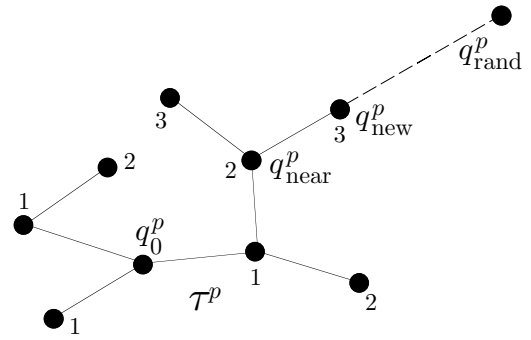

Fig. 3. The tree expansion in the subspace of platform configurations. The integer $k$ associated to each node identifies the end-effector pose $p_{k}$ of which the node is a preimage. Note that the path between adjacent configurations, represented here as a segment for simplicity, is by construction feasible w.r.t. the nonholonomic constraints.

identifies $q_{\text {near }}$, the node of $\tau$ closest $^{7}$ to $q_{\text {rand }}$ with respect to the platform variables, and returns the index $k$ of the end-effector pose $p_{k}$ to which $q_{\text {near }}$ is associated. Then, RAND_CONF computes $q_{\text {new }}$ by generating first a random input (using one of the strategies described in Sect. III-B), then the corresponding $q_{\text {new }}^{p}$ starting from $q_{\text {near }}^{p}$, and finally (if successful) $q_{\text {new }}^{m}$ by inverse kinematics on the manifold associated to $p_{k+1}$. The path joining $q_{\text {near }}$ to $q_{\text {new }}$ is now checked for collision; if the result is negative, $\tau$ is expanded and $p_{i+1}$ is returned. Figure 3 shows the expansion of the tree $\tau$ in the subspace of platform configurations.

The role of $q_{\text {rand }}$ in guiding the expansion of RRT_LIKE is only to identify $q_{\text {near }}$, the closest node to $q_{\text {rand }}$ w.r.t. the platform variables; the direction of expansion from $q_{\text {near }}$ is then determined by the choice of the pseudovelocity input, and in general does not depend on $q_{\text {rand }}$. This is quite different from what happens in the classical RRT algorithm, where the direction of expansion is chosen to be the line joining $q_{\text {rand }}$ with $q_{\text {near }}$. To retain this strategy, which is indeed essential for the effectiveness of RRT (namely, for driving the expansion toward wide Voronoi regions), one can adopt the optimal pseudovelocity generation outlined in Sect.III-B, using the $I_{\text {dist }}$ criterion with $q_{\text {des }}=q_{\text {rand }}$.

\section{Variations on RRT_LIKE}

The expansion of $\tau$ toward randomly selected directions gives to RRT_LIKE an exploratory attitude which, for the MPEP problem, could prove inefficient due to the strong constraint represented by the end-effector path. However, it is possible to modify the RRT_LIKE planner by alternating depth-first searches with expansion steps. This can be done by invoking the STEP function right after the EXTEND_LIKE operation has been executed; the arguments passed to STEP are $p_{l}, p_{s}$, where $p_{l}$ is the closest pose to $p_{s}$ reached so far by the algorithm. This modified RRT-based planner, which tries at the same time to explore the portion of configuration space consistent with the end-effector path constraint and to approach the goal self-motion manifold through a greedy search, is called RRT_GREEDY.

The exploratory attitude of RRT_LIKE may also be a drawback when the start and goal self-motion manifolds are very distant. Inspired by [12], one possible solution is

\footnotetext{
${ }^{7}$ Here, we use a weighted euclidean metric for simplicity, but a more appropriate nonholonomic metric can be adopted.
}

to expand two trees, respectively rooted at the start and at the goal self-motion manifolds. The trees will 'meet' on some intermediate manifold, but different nodes will be generated; it is then necessary to compute a self-motion connecting the two nodes by an RRT-based search restricted to the manifold. This planner is called RRT_BIDIR.

\section{Planning Experiments}

We now present some MPEP experiments for the mobile manipulator of Fig. 1. The algorithms were implemented in $\mathrm{C}$ on a $1 \mathrm{Ghz} \mathrm{PC}$ and integrated in the software platform Move3D, dedicated to motion planning and developed at LAAS-CNRS, France ${ }^{8}$.

The first two experiments aim at highlighting the effects of the different pseudovelocity generation procedures of Sect.III-B: to this end, the mobile manipulator must move its end-effector along a given rectilinear path in the absence of obstacles, and only the RRT_LIKE planner is used.

For the first experiment, Fig. 4 compares the solution tree (more precisely, its projection on the $x, y$ plane) obtained by completely random pseudovelocity inputs (left) and constant-energy optimal inputs with criterion $I_{\text {dist }}$ (right). The left tree is uselessly erratic (given that no obstacles are present) if compared with the right one, which extends mostly along the direction of the task trajectory. The table reports a performance comparison (averaged over 20 trials) between these two and other methods, in terms of time needed to find a solution, failures of INV_KIN, and nodes in the tree. The superiority of constant-energy methods is confirmed by the smaller number of kinematic inversion failures and by the reduced tree size. As for the choice of the performance criteria, the minimization of $I_{\text {dist }}$ appears to be more effective than the maximization of $I_{\text {comp }}$. $I_{\text {mix }}$ denotes a weighted criterion that combines the two.

The second experiment is similar to the first, but a different initial configuration has been assigned. In particular, the arm is completely stretched and the mobile platform orientation is orthogonal to the end-effector path; hence, $q_{0}$ has a low task compatibility with respect to the given end-effector trajectory. Figure 5 shows the results obtained by RRT_LIKE using constant-energy optimal inputs with the performance criteria $I_{\text {dist }}$ (left) and $I_{\text {comp }}$ (right). In this case, the use of $I_{\text {comp }}$ allows the robot to recover and maintain a higher compatibility value (note the absence of kinematic inversion failures), ultimately resulting in a smoother motion and in a smaller computation time.

Experiments of the second group take place in environments with obstacles, and aim at comparing the performance of the various planners. In these trials, pseudovelocity inputs are generated by optimizing the mixed performance criterion $I_{\text {mix }}$ over a set of four constant-energy candidates in $\mathcal{U}_{1}, \ldots, \mathcal{U}_{4}$ (see Sect. III-B). As before, the planners' performances are averaged over 20 trials.

The third experiment scene is quite simple. The robot must move its end-effector along a polynomial path through

\footnotetext{
${ }^{8}$ Move3D is at the origin of the product KineoWorks currently marketed by the company Kineo CAM (www.kineocam.com).
} 


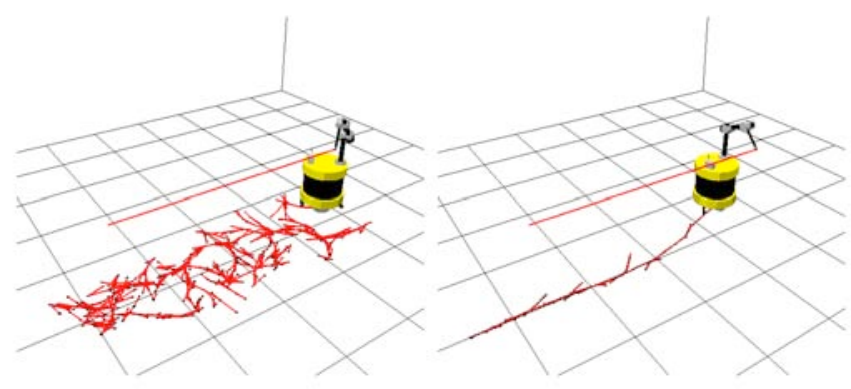

\begin{tabular}{|c||r|r|r|}
\hline Pseudovelocity generation & time $(\mathrm{s})$ & \# kin fail & \# nodes \\
\hline \hline completely random & 2.16 & 41 & 165 \\
constant energy & 0.50 & 17 & 127 \\
optimal (comp. rand., $I_{\text {dist }}$ ) & 2.00 & 59 & 158 \\
optimal (const. ene., $I_{\text {dist }}$ ) & 0.33 & 4 & 96 \\
optimal (const. ene., $I_{\text {comp }}$ ) & 1.00 & 10 & 189 \\
optimal (const. ene., $I_{\text {mix }}$ ) & 0.60 & 21 & 115 \\
\hline
\end{tabular}

Fig. 4. First experiment: The tree built on the $x, y$ plane by RRT_LIKE using completely random inputs (left) and constant-energy optimal inputs with criterion $I_{\text {dist }}$ (right). Also reported is a comparison extended to other input generation methods.
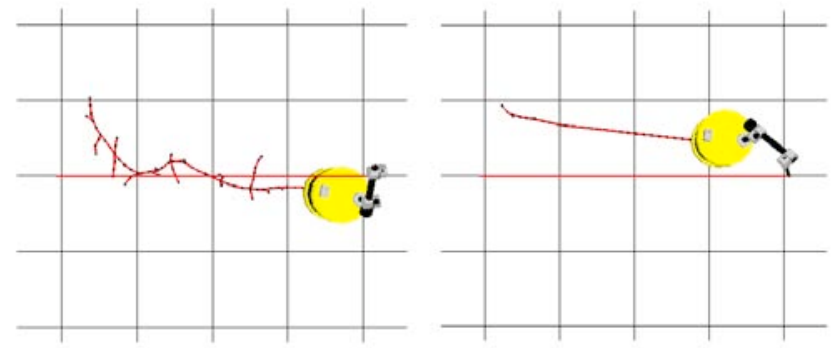

\begin{tabular}{|c||r|r|r|}
\hline Criterion & time (s) & \# kin fail & \# nodes \\
\hline \hline$I_{\text {dist }}$ & 0.90 & 145 & 78 \\
$I_{\text {comp }}$ & 0.64 & 0 & 55 \\
\hline
\end{tabular}

Fig. 5. Second experiment: The tree built on the $x, y$ plane by RRT_LIKE using constant-energy optimal inputs with criteria $I_{\text {dist }}$ (left) and $I_{\text {comp }}$ (right), with a comparison between the two methods

two columns. Figure 6 contains some frames from the solution path computed by GREEDY and a table summarizing the performance of the planners. In this case, the GREEDY planner performs best under all aspects due to its depth-first strategy, which is invariably more effective in easy planning problems. Also RRT_BIDIR achieves a good result thanks to the large space available for reconfiguration.

Figure 7 shows the scene of the fourth experiment; the end-effector must follow a rectilinear path which is dangerously close to an obstacle. The solution shown was planned by RRT_GREEDY; note how the robot stretches the arm to move under the obstacle. RRT-based algorithms perform best in this case thanks to their exploratory attitude, with the exception of RRT_BIDIR, which is penalized by the reduced space for reconfiguration in the contact manifold.

The fifth planning problem is very difficult: to complete its task, the mobile manipulator must first cross a narrow passage and then carefully move its arm so as to drive the end-effector between the sandwich-shaped obstacle. While GREEDY failed to produce a solution within the allotted time, RRT-based planners performed quite well.

A number of experiments have confirmed the above

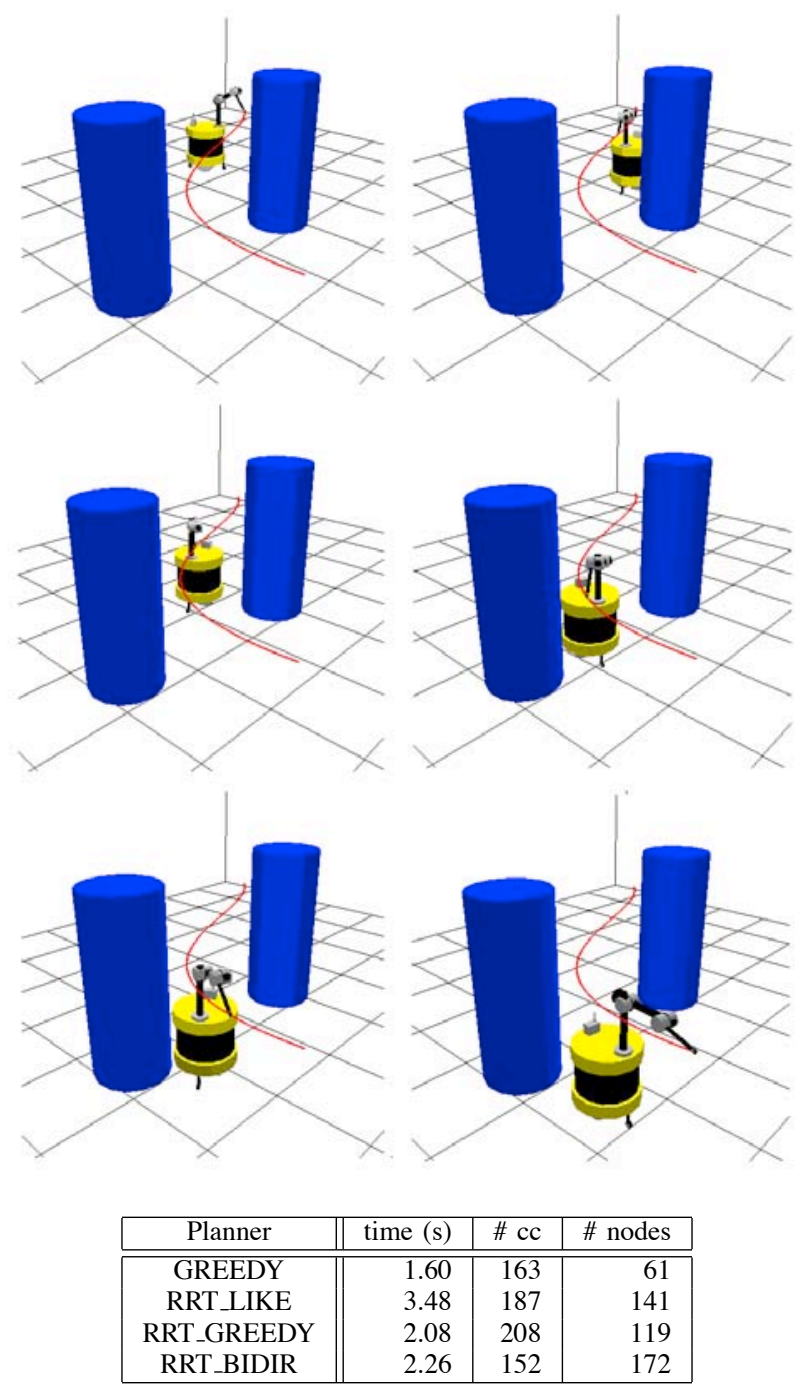

Fig. 6. Third experiment: Solution obtained with GREEDY (left to right, and top to bottom) and comparison of planners' performance

indications. Essentially, GREEDY is very effective when dealing with simple queries, while RRT_LIKE and RRT_GREEDY perform much better when the difficulty of the problem increases. The bidirectional strategy of RRT_BIDIR is convenient when there is a large space available for reconfiguration, as in the case of Fig. 9.

\section{CONCLUSIONS}

Single-query probabilistic planners have been presented for the problem of generating collision-free motions for a nonholonomic mobile manipulator moving along a given end-effector path. Experiments show that simple instances of the problem can be solved more efficiently by a greedy approach, whereas the breadth-first search of RRT-based planners is needed to deal with more complex cases.

Among the issues deserving further attention, we mention $(i)$ proving probabilistic completeness along the lines of [8,11], (ii) complexity analysis, and (iii) the choice of performance criteria for pseudovelocity generation, with the ideas in [13] as possible inspiration. An extension of the proposed methods to sensor-based exploration can also be envisaged following the approach in [14]. 

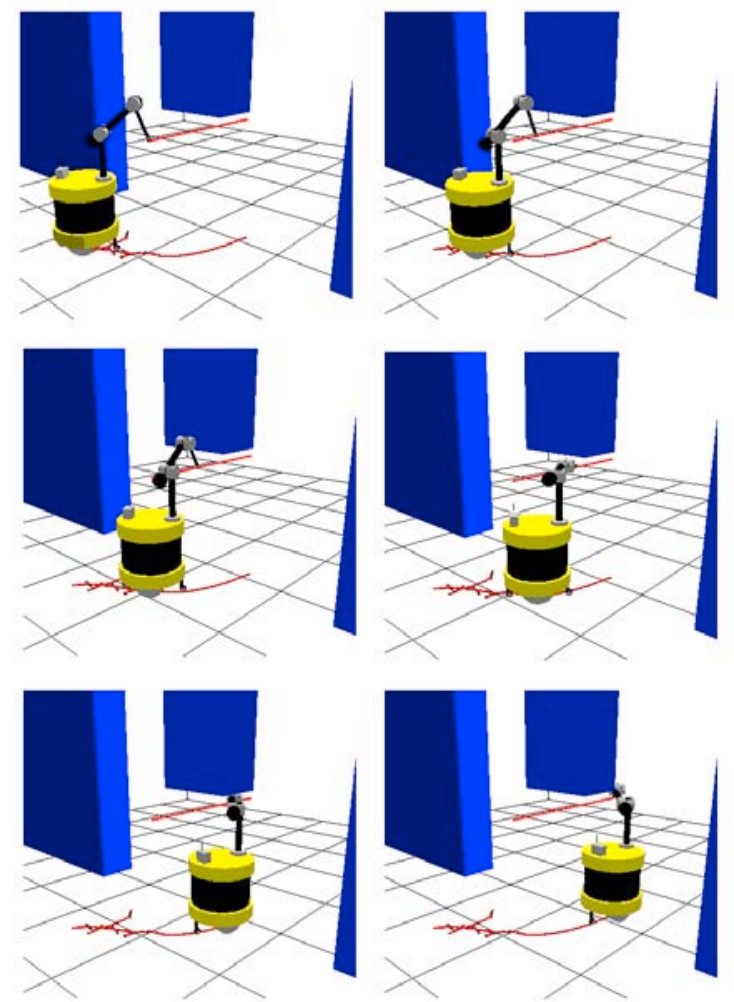

\begin{tabular}{|c||r|r|r|}
\hline Planner & time (s) & \# CC & \# nodes \\
\hline \hline GREEDY & 12.6 & 478 & 40 \\
RRT_LIKE & 6.3 & 218 & 115 \\
RRT_GREEDY & 6 & 278 & 76 \\
RRT_BIDIR & 33 & 1230 & 511 \\
\hline
\end{tabular}

Fig. 7. Fourth experiment: Solution obtained with RRT_GREEDY and comparison of planners' performance

\section{REFERENCES}

[1] D. Apostolopoulus, M. Wagner, and W. Whittaker, "Technology and field demonstration results in the robotic search for antarctic meteorites," Field and Service Robotics Conf., 1999.

[2] M. Nechyba and Y. Xu, "Human robot coordination in space: (sm)^2 for new space station structure," IEEE Robotics and Automation Mag., vol. 2, no. 4, pp. 4-14, 1995.

[3] Y. Yamamoto and X. Yun, "Coordinating locomotion and manipulation of a mobile manipulator," IEEE Trans. on Automatic Control, vol. 39, pp. 1326-1332, 1994.

[4] B. Siciliano, "Kinematic control of redundant robot manipulators: A tutorial," J. of Intelligent and Robotic Systems, vol. 3, pp. 201-212, 1990.

[5] D. P. Martin, J. Baillieul, and J.M. Hollerbach, "Resolution of kinematic redundancy using optimization techniques," IEEE Trans. on Robotics and Automation, vol. 5, pp. 529-533, 1989.

[6] A. Mohri, S. Furuno, and M. Yamamoto, "Trajectory planning of mobile manipulator with end-effector's specified path," 2001 IEEE Int. Conf. on Intelligent Robots and Systems, vol. 4, pp. 2264-2269, 2001.

[7] G. Oriolo, M. Ottavi, and M. Vendittelli, "Probabilistic motion planning for redundant robots along given end-effector paths," 2002 IEEE Int. Conf. on Intelligent Robots and Systems, vol. 2, pp. 16571662, 2002.

[8] L. Han and N. Amato, "A kinematic-based probabilistic roadmap method for closed chain systems," 4th Int. Work. on Algorithmic Foundations of Robotics, pp. 233-246, 2000.

[9] J. Cortes, T. Simèon, and J. P. Laumond, "A random loop generator for planning the motions of closed kinematic chains using prm methods," 2002 IEEE Int. Conf. on Robotics and Automation, pp. 2141-2146, 2002.

[10] S. L Chiu, "Task compatibility of manipulator postures," The Int. J. of Robotics Research, vol. 7, pp. 13-21, 1988.
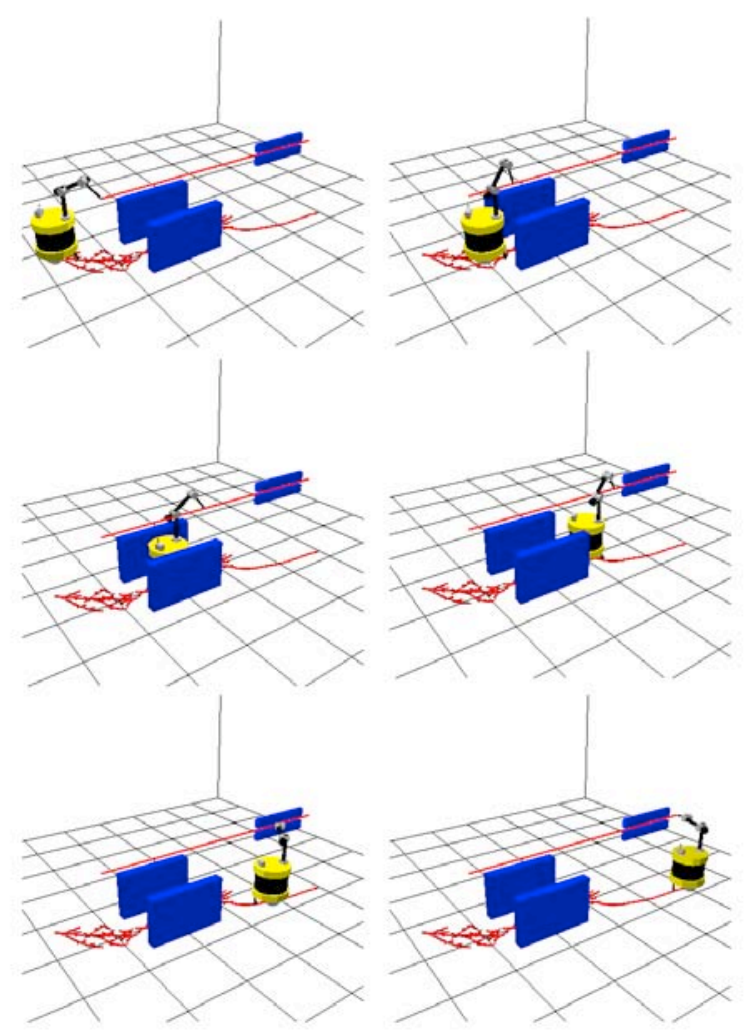

\begin{tabular}{|c||r|r|r|}
\hline Planner & time (s) & \# cc & \# nodes \\
\hline \hline GREEDY & - & - & - \\
RRT_LIKE & 33 & 1768 & 560 \\
RRT_GREEDY & 47 & 5439 & 229 \\
RRT_BIDIR & 230 & 15769 & 1322 \\
\hline
\end{tabular}

Fig. 8. Fifth experiment: Solution obtained with RRT_LIKE and comparison of planners' performance

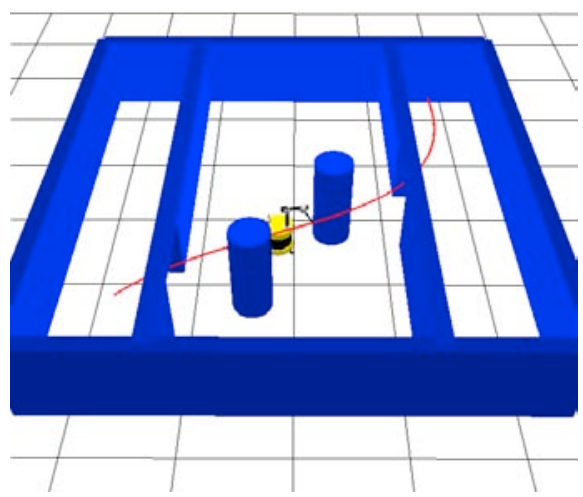

Fig. 9. A problem for which RRT_BIDIR is more efficient than other planners

[11] S. M. LaValle, "Rapidly-exploring random trees: A new tool for path planning," Technical Report No. 98-11, Computer Science Dept., Iowa State University., 1998.

[12] J. J. Kuffner and S. M. LaValle, "Rrt-connect: An efficient approach to single-query path planning," 2000 IEEE Int. Conf. on Robotics and Automation, vol. 2, pp. 995-1001, 2000.

[13] P. Leven and S. Hutchinson, "Using manipulability to bias sampling during the construction of probabilistic roadmaps," IEEE Trans. on Robotics and Automation, vol. 19, pp. 1020-1026, 2003.

[14] G. Oriolo, M. Vendittelli, L. Freda, and G. Troso, "The srt method: Randomized strategies for exploration," 2004 IEEE Int. Conf. on Robotics and Automation, pp. 4688-4694, 2004. 